\title{
Utilização detécnicas de jogos para a análise visual de informações
}

\author{
Use of gaming techniques for visual information analysis
}

\author{
El uso de técnicas de juego para elanálisis de lainformación visual \\ Hudson Rodrigues Costa \\ Rosalvo Ermes Streit ${ }^{2}$ \\ http://dx.doi.org/10.5216/32365
}

\section{Resumo}

O excesso de informações e a abundância de dados, com diversidade na forma e no conteúdo,tornam os processos de tomada de decisão mais complexos. Nesse contexto, a apresentação visual das informações tem se mostrado uma alternativa interessante de apoio aos gestores e especialistas. Contudo, é necessário que as interfaces sejam adequadas para facilitar e promover a análise das informações. Nesse contexto, este artigo descreve pesquisas que utilizam o conceito dejogos. Explorase a importância da gestão da informação, a utilização do gamification para a análise visual de informações, bem como as motivações psicológicas relacionadas às mecânicas de jogos no processo decisório. Em função da baixa quantidade de estudos sobre o emprego de gamification nessa área, o artigo traz reflexões relevantes sobre o tema.

Palavras-chave: Técnicas de jogos.Gamification. Interação homem-maquina (HCI). Análise visual de informações. Processo decisório.

\begin{abstract}
Information overload and abundance data, with diversity in form and content, make the decision making processes more complex. On the other hand, the visual presentation of information has been an interesting alternative to support managers and specialists. However, it is necessary that the interfaces are appropriate to facilitate and promote the analysis of the information. In this context, this article describes research that uses the concept of games. It explores the importance of information management, the use of gamification for visual information analysis, as well as the psychological motivations related to mechanical games in decision making process. Due to the low number of studies about gamification in this area, the article presents relevant thoughts on the subject.
\end{abstract}

Keywords: Game techniques. Gamification. Human-computer interaction (HCI). Visual analytics. Decision process.

\section{Resumen}

La sobrecarga de información y datos de abundancia, con la diversidad en forma y contenido, hacen más complejo el proceso de toma de decisiones. Por otra parte, la presentación visual de la información ha sido una alternativa interesante para apoyar a los directivos y especialistas. Sin embargo, es necesario que las interfaces son apropiadas para facilitar y promover el análisis de la información. En este contexto, el presente artículo se describen investigaciones utilizando concepto de juegos. Explora la importancia de la gestión de la información, el uso de la gamificación para el análisis de la información visual, así como las motivaciones psicológicas relacionadas con las

\footnotetext{
${ }^{1}$ Mestrando do Programa de Pós-Graduação Stricto Sensu em Gestão do Conhecimento e da Tecnologia da Informação (MGCTI), na Universidade Católica de Brasília (UCB). Brasil, Brasília, DF. Email:hudsonrc@hotmail.com

${ }^{2}$ Doutor pela Universidade Federal do Rio Grande do Sul (UFRGS), Mestre em Ciência da Computação pela Universidade Federal do Rio Grande do Sul (UFRGS). Professor do Mestrado em Gestão do Conhecimento e Tecnologia da Informação (MGCTI) da Universidade Católica de Brasília (UCB). Brasil, Brasília, DF. E-mail: rosalvo.streit@gmail.com
}

Comun. \& Inf., Goiânia, GO, v. 18, n. 1, p. 110-123, jan./jun. 2015 
mecânicas de los juegos en proceso de toma de decisiones. Debido al bajo número de estudios sobre gamificación en esta área, el artículo presenta reflexiones pertinentes sobre el tema.

Palabras clave: Técnicas de juego. Gamificación. Interacción persona-ordenador (HCI). Analítica visual. Proceso de decisión.

\section{INTRODUÇÃO}

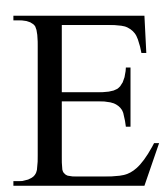

$\mathrm{m}$ tempos de disponibilidade de meios físicos e virtuais para armazenamento e transmissão digital de dados mais acessíveis e sofisticados, a capacidade de retenção e transporte de dados tornam-se cada vez mais fáceis.Contudo, transformar dados em informação, para a devida e tempestiva tomada de decisão, torna-se, a cada dia, não só missão crítica, mas sim, vital às organizações públicas e privadas. Neste contexto, a gestão da informação (GI) é um instrumento indispensável para balancear o uso das informações com os recursos corretos para suporte à tomada de decisão.

De acordo com Davenport e Prusak(1997), GI é um conjunto estruturado de atividades que espelha a forma pela qual uma organização captura, distribuie usa a informação e o conhecimento para obter mais eficiência no uso dos dados e informações organizacionais.

Alguns pesquisadores estão trabalhando em métodos que aprimorem a apresentação do grande volume de informações. Segundo Ebert, Gershon e Veer(2012), a interação homem máquina (do inglês, human-computerinteraction - HCI) é um termo conhecido desde o início de 1980 que está relacionado ao aprimoramento da interação entre usuários e dispositivos de computação, de forma a adequar a apresentação das informações, tornando-as mais adaptadas às necessidades do negócio e, consequentemente, para a tomada de decisão.

A partir das pesquisas relacionadas à psicologia cognitiva, que é uma das disciplinas base de HCI, surgem estudos que avaliam a aplicação de técnicas de design de jogos em situações que não são jogos. As técnicas de jogos, em contexto que não são jogos, são incrementadas pela motivação, concentração, esforço e colaboração entre os envolvidos da organização. A ideia é tornar as aplicações mais atrativas para o usuário. Desta forma, surge a teoria da diversão (do inglês,funologyougamification) (WERBACH eHUNTER, 2012;CUNHINGHAM eZICHERMANN, 2011).

Considerandoo contexto apresentado, o fator motivador deste artigo é explorar, nas bases de publicações científicas, livros, teses e dissertações, a utilização de métodos de jogos que direcionem o comportamento do usuário a estratégias de tomada de decisão baseadas em informações.Assim, investiga-se o uso degamificationpara a análisevisual deinformações, de modo que o usuário aumente o seu envolvimento com os dados e aprimore, desse modo, a sua tomada de decisão.

Comun. \& Inf., Goiânia, GO, v. 18, n. 1, p. 110-123, jan./jun. 2015 
Por meio destarevisão literária, é abordada a importância da gestão da informação e o uso da análise visual para a tomada de decisão. Em seguida,descrevem-se os estudos relacionados àinteração homem-máquina com referência à usabilidade,ao design centrado no ser humano e a maneira como a visualização das informações pode influenciar o seu fluxo e distribuição. Após, abordam-se a utilização de mecanismos de jogos em contextos que não jogos e as motivações psicológicas que os elementos do gamification podem exercer sobre os usuários no âmbito de processos decisórios.Por fim, discutem-se os principais aspectos dos sistemas colaborativos de apoio à decisão e um exemplo de pesquisa aplicada sobre uma solução de gamification em contexto de tomada de decisões por meio de brainstorming.

\section{REVISÃOBIBLIOGRÁFICA}

\subsection{GESTÃO DA INFORMAÇÃO}

O gerenciamento da informaçãoé um conjunto estruturado de atividades que espelha a forma pela qual uma organização captura, distribui, e usa informação e conhecimento (DAVENPORT e PRUSAK, 1997). Conforme Wilson (1999), a gestão inadequada de informações pode gerar diversos problemas: de uma simples ineficiência até uma responsabilidade legal, ou mesmo um escândalo. A informação, deste modo, deve ser administrada da mesma forma que tradicionalmente gerenciam-se outros ativos, como financeiro, recursos humanos e materiais (MIRANDA e STREIT, 2007).

A gestão da informação (GI) nas organizações é fator crítico de apoio à devida e tempestiva tomada de decisão. Observa-se na literatura que os estudos que tratam do uso da informação no processo decisório são amplos e multidisciplinares. Segundo Kennerley e Mason (2008), a área de gestão contribui consideravelmente para esse assunto, assim como outras disciplinas nas áreas de ciências sociais, tecnologia da informação e psicologia. De acordo com esses autores, não há consenso sobre os mecanismos e abordagens relacionadas à tomada de decisão. Apesar disso, os resultados da pesquisa de Goodman (1993) deixam claro que o sucesso dos gestores depende do uso efetivo das informações.

Contudo, o advento de computadores com grande poder de processamento e a disponibilidade de vastos volumes de dados geram desafios aos gestores e organizações para a análise e exploração dessas informações. Por outro lado, esse ambiente potencializa a criação de novas informações e conhecimentos mediante o uso de técnicas de visualização, organizando conjuntos de dados complexos em modelos simplificados que apresentem novas 
perspectivas de percepção aos novatos e especialistas (MILLER et al., 2012). Segundo Sawant e Healey (2007), a visualização é uma área da computação gráfica que gerencia e apresenta informação na forma visual para facilitar de forma rápida e efetiva a sua análise e interpretação.Neste contexto, onde a GI e os SI apoiam a tomada de decisão, observa-se que o uso da análise visual de dados (do inglês, Visual Analytics)contribui significativamente para dispor a informação de forma mais compreensível.Definido por Thomas e Cook (2005, p.4)como "a ciência da análise do raciocínio suportado por interfaces visuais interativas", a análise visual de dados oferece uma perspectiva científica sobre a interação humana com interfaces gráficas complexas. Seu objetivo é apoiar a tomada de decisão humana em situações caracterizadas como problemáticas em diversos domínios de aplicação.

Segundo Goodman (1993), a disponibilidade de tecnologias avançadas de informação aumenta a comunicação e as opções para a tomada de decisão, bem como aprimoram a qualidade dessas decisões. De acordo com o autor, uma maior facilidade para a compreensão e acesso às informações, aumenta a responsabilidade dos gestores para o seu uso e, consequentemente, promove melhores tomadas de decisões.

Neste contexto, a visão analítica contribui na interação homem-máquina, (do inglês, Human Computer Interaction - HCI), que trata do desempenho e da tarefa cognitiva de como ela afeta a interpretação das informações, visualização e apresentação dos dados. A relação entre a tomada de decisão e análise cognitiva da informação emprega esforços para criar software e soluções que maximiza a capacidade humana de perceber, compreender e raciocinar sobre dados e situações complexas e dinâmicas (GOODMAN, 1993).

Os autores pesquisados associam a noção do HCI com a importância da interação visual dos sistemas de informação e a sua relação com as disciplinas da ciência da computação e da psicologia humana.Nesse contexto, segundoDeterding (2012), o campo de estudo relacionado ao HCI começa a explorar várias facetas da experiência do usuárioe projetos relacionados ao prazer, diversão e motivação.Nessa área, o interesse pelo termo gamification tem sido crescente ${ }^{3}$.

\subsection{GAMIFICATION}

A noção de gamification pode ser compreendida como a utilização de mecanismos de jogos em contextos que não são jogos (DETERDING, 2011; WERBACH e HUNTE, 2012; CUNNINGHAM e ZICHERMAN, 2011). Uma das características dessa técnica é motivar,

\footnotetext{
${ }^{3}$ É possível observar o crescimento do termo gamification no Google Trends, que possibilita verificar as buscas por determinados termos em um passado recente e suas tendências (http://www.google.com/trends/).
}

Comun. \& Inf., Goiânia, GO, v. 18, n. 1, p. 110-123, jan./jun. 2015 


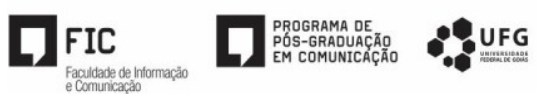

incentivar e orientar os usuários mediante o desenvolvimento de interfaces visuais com modelos que transmitem experiências equivalentes aos princípios e elementos utilizados em jogos.

De acordo com Hägglund (2012) e Morrison (2014),o termo gamificationdeve ter sido utilizado pela primeira vez por Nick Pelling, uma programadora de jogos de origem britânica, que batizou a sua empresa de consultoria com o termo "gamificationconsultancy" (PELLING, 2014). A consultoria de Pelling inventou e criou uma série de jogos de computadores na década de 1980 para a BBC Micro, a máquina usada pela maioria escolas no Reino Unido na época (MORRISON, 2014). Sua ideia era ajudar as empresas a evoluir seus produtos em plataformas de entretenimento. Pelling, possuía a visão de que todos os dispositivos em breve se tornariam um jogo.

Conforme já mencionado, a principal característica do conceito gamification é a utilização de mecânicas de jogos em contextos convencionais, ou seja, que não são jogos. De acordo com Cook (2006), mecânicas de jogos são sistemasbaseados em regrasousimulações que facilitam e incentivam os usuários na exploração e aprendizagem através do uso de mecanismos de retroalimentação (do inglês, feedback mechanisms).

De acordo com Ferro, Walz e Greuter (2013), a utilização de mecanismos e elementos de jogos para a apresentação das informações de sistemas computacionais podem influenciar a criação, troca e distribuição dessas informações. As reações podem ser ativadas ou estimuladas com elementos de jogos, tais como: emblemas, placas de líder, metas, personagens virtuais (do inglês, avatars), que podem provocar a competição e a autoestima dos usuários (jogadores). Dada a diversidade dos tipos de usuários com diferentes características, podem-se utilizar diferentes elementos de jogos para estimular, de forma adequada, o comportamento do público-alvo e o fluxo das informações de interesse (FERRO, WALZ eGREUTER, 2013).

Segundo Marczewski (2013),ogamification é, normalmente, utilizado com o objetivo de influenciar comportamentosdesejáveis nos indivíduosdurante a execução de atividades emprocessos de trabalho e no uso de sistemas de informação. Assim, o uso dessa técnicapode tornar a tecnologia mais envolvente, ajudando na solução de problemas e incentivando os usuários a interagir com determinados comportamentos do sistema, devido a pré-disposição psicológica dos seres humanos em lidar com jogos e suas mecânicas. 


\subsubsection{Exemplos de sistemas e aplicações de Gamification}

Para tornar as atividades convencionaismais divertidas e influentes, diversas indústrias de desenvolvimento de sistemas de informação e aplicativos aplicam técnicas de gamification,nas mais variadas situações. As corporações também têm aplicado métodos e sistemas que utilizam gamification para incentivar, reter,influenciar a colaboração e a participação dos funcionários. No Quadro 1, são apresentadosalguns exemplos de aplicação de métodos de gamificationem diversas organizações.

Quadro 1 - Exemplos de sistemas e aplicações degamification nas empresas

\begin{tabular}{|c|c|c|}
\hline Sistema & Descrição & Empresas \\
\hline Badgeville & $\begin{array}{l}\text { Oferece um conjunto de produtos que ajudam } \\
\text { as empresas a medir e a influenciar os } \\
\text { comportamentos dosusuários através das } \\
\text { experiências que empregados e clientes } \\
\text { experimentam. }\end{array}$ & $\begin{array}{l}\text { Samsung, Dell, Deloitte, } \\
\text { eBay, Fox, Recyclebank, } \\
\text { Active Network, e CA } \\
\text { Technologies. }\end{array}$ \\
\hline $\begin{array}{l}\text { Big Door } \\
\text { Media }\end{array}$ & $\begin{array}{l}\text { A plataforma oferece pequenos códigos de } \\
\text { recursos gamificationque são embutidos em } \\
\text { sítios da internet. Esses componentes } \\
\text { oferecemrecompensas (selos, medalhas, etc) } \\
\text { aos usuários que alcançam metas e objetivos } \\
\text { específicos. }\end{array}$ & $\begin{array}{l}\text { Dell, MLB.com, } \\
\text { Nickelodeon, } \\
\text { Wetpaint and music artists } \\
\text { Big K.R.I.T. e 2-Chainz. }\end{array}$ \\
\hline Bunchball & $\begin{array}{l}\text { Este sistema permite a criação, de forma fácil, } \\
\text { de regras, recompensas, itens virtuais, } \\
\text { notificações que podem ser publicadas } \\
\text { instantaneamente em redes sociais, grupos e } \\
\text { demais redes de colaborações virtuais } \\
\text { (conteúdos de propriedade digital). }\end{array}$ & $\begin{array}{l}\text { Bravo, Campusfood.com, } \\
\text { Chiquita, Playboy, } \\
\text { Rightguard, } \\
\text { LiveOps, MTV, NBC, Syfy, } \\
\text { USA Network, Telemundo, } \\
\text { Warner Bros. e Wendy's }\end{array}$ \\
\hline Gigya & $\begin{array}{l}\text { A empresa está emgamificationde consumo. } \\
\text { Gigyadesenvolve as funcionalidades viaplug- } \\
\text { ins interoperáveis com os produtos da empresa. } \\
\text { Esses componentes têm características de } \\
\text { gamification, como, por exemplo: } \\
\text { classificações (leaderboards), perfis de usuário, } \\
\text { conquistas e recompensas,notificações, } \\
\text { informações das atividades de notícias do } \\
\text { usuário (feeds). }\end{array}$ & $\begin{array}{l}\text { ABC, American Express, } \\
\text { CBS, Chrysler, Cnet, CNN, } \\
\text { Comcast, E!, G4, HP, Los } \\
\text { Angeles Times, Microsoft. } \\
\text { MSN, NBA, NBC, Fox, } \\
\text { Reuters, Rubbermaid, } \\
\text { Sprint, Starz, Tesla Motors, } \\
\text { Time, Time Warner Cable, } \\
\text { TNT e Universal. }\end{array}$ \\
\hline
\end{tabular}

Fonte: Meloni et al. (2012), adaptado pelos autores.

Além dos exemplos apresentados acima, cabe destacar que grande parte dos estudos de gamificationtêm sido realizada na área da educação. A Khan Academyutiliza técnicas degamificationpara motivar a educação e o aprendizado dos seus usuários (KHANACADEMY, 2014).Conforme Morrison et al. (2014), a Khan Academy possui aproximadamente 15 milhões de usuários registrados. O portal de aprendizado utiliza 
elementos de motivação e engajamento a curto prazo e procura usar gamification para manter alunos envolvidos nas tarefas mais difíceis.

Outro exemplo é a Codecademy,objetivo é utilizar de maneira inteligente ogamificationpara tornar o aprendizado divertido(CODECADEMY, 2014). Assim como a Khan Academy, a Codecademy tem recebido grande atenção e publicidade devido ao uso de técnicas de gamification, tornando as atividades dos sítios divertidas de serem realizadas (HÄGGLUND, 2012).

\subsubsection{Tendências da utilização de Gamification}

De acordo o relatório elaborado pela consultoria Gartner (2011), as estimativas são de que em 2015 mais de $50 \%$ das organizações que gerenciam processos de inovação irão utilizar métodos de gamification. A consultoria M2-Research (MELONI et al., 2012), através de suas pesquisas,demonstraque o crescimento do mercado de gamification é consistente, conforme o Gráfico 1. As projeções globais da M2 Research (MELONI et al., 2012) consideram que em 2016 a tecnologia de gamificationpoderá alcançar a receita de 2.8 bilhões de dólares.

Gráfico 1 - Previsão do mercado de gamification

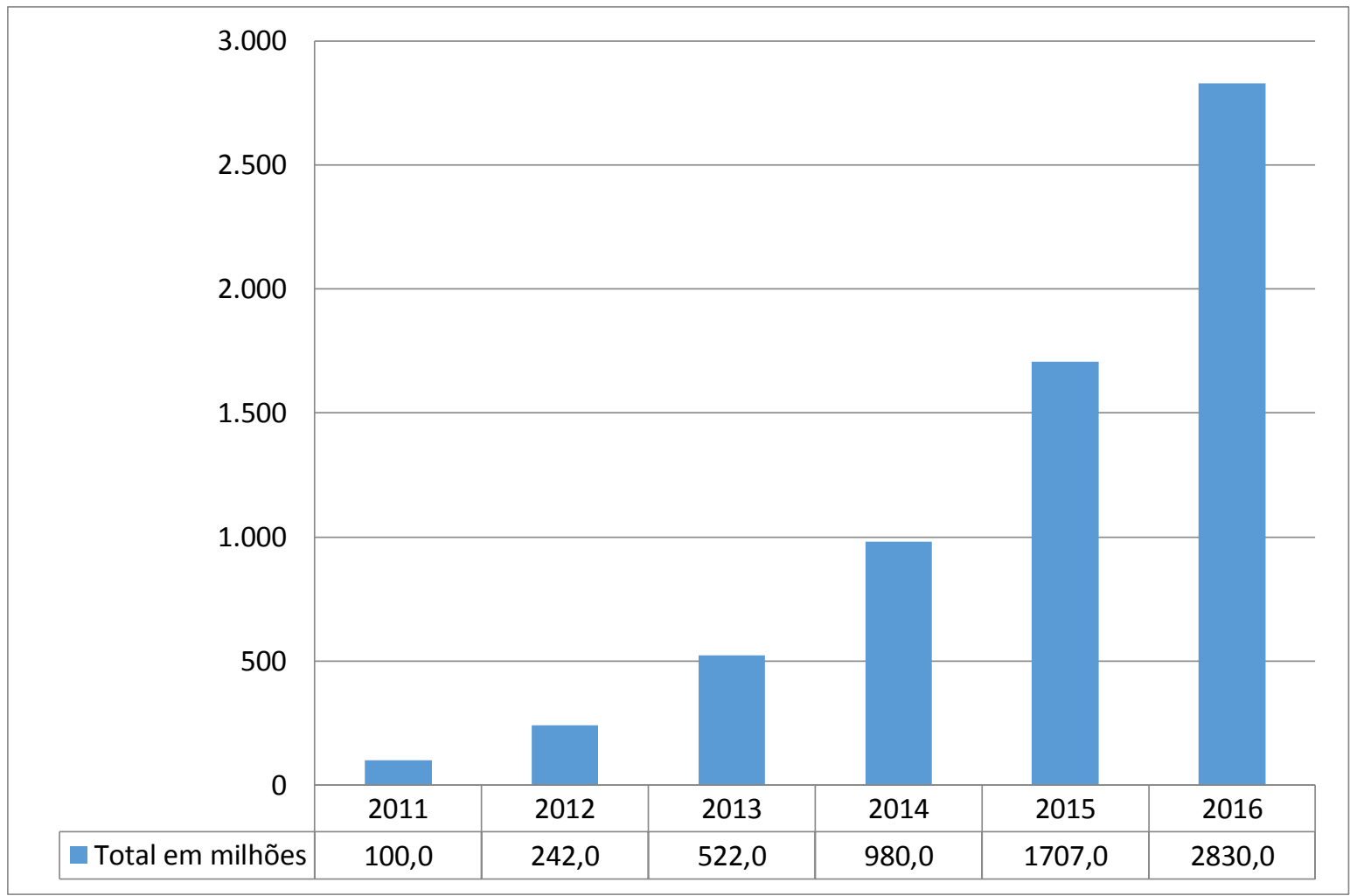

Fonte: Meloni et al. (2012), traduzido pelos autores. 


\subsubsection{Sistemas de tomada de decisão com Gamification}

O desenvolvimento de funcionalidades que suportem o processo de tomada de decisão é fator relevante na concepção dos sistemas de informação. Contudo, segundo Sanfilippo et al. (2013), a sua implementação em sistemas computacionais é difícil, pois o processo de tomada de decisão é fortemente estimulado pela percepção e criatividade, e influenciado por vieses cognitivos.Apesar disso, alguns pesquisadores têm voltado a sua atenção a novas ferramentas de apoio à tomada de decisão, combinando recursos de colaboração social com técnicas de estruturação e de análise das decisões (KARACAPILIDIS e PAPADIAS, 2001; IANDOLI et al., 2008).

Moradian et al. (2014), da Universidade de Toronto, por exemplo, projetaram uma Interface de Programação de Aplicativos (do inglês, ApplicationProgramming Interface $A P I)$ para redes sociais, desenvolvida pela empresa Google (Google OpenSocial), integrada com a ferramenta de tomada de decisão colaborativa chamada SAP StreamWork, aplicando mecanismos de gamification. ConformeKreamer et al.(1988), os sistemas de apoio à decisão de forma colaborativa (do inglês, Collaborativedecisionsupport systems- CDSS), sãodefinidos como sistemas de suporte a decisão de forma colaborativa e participativa que facilitam a solução de problemas estruturados por um conjunto de decisores, aumentando a eficácia das decisõespor meio do compartilhamento interativo de informações entre os membros.

OSAP StreamWork é um ambiente de tomada de decisão colaborativa hospedado na Internet que permite a tomada de decisões em conjunto, por meio de discussão dos assuntos e compartilhamento de informações confiáveis, bem como a coordenação de pessoas e o monitoramentodedebate de ideias (do inglês, brainstorming), promovendo tomada de decisões com base em fatos e evidências (SAP, 2014). O objetivo da pesquisa realizada pelos cientistas da Universidade de Toronto foi desenvolver uma solução gamificationque motivassea participação dos usuários na tomada de decisões mediantebrainstorming colaborativo (debates,segmentação de grupos de sugestões, convergênciadas melhores ideias para novos ciclos dediscussãoentre os participantes). $\mathrm{O}$ resultado possibilita a visualização da informação de maneira estruturada e segmentada, oferecendo as melhores opções para a tomada de decisão pelos decisores(MORADIAN et al., 2014).

Para a solução, os autores utilizaram elementos relacionados a jogos, tais como,definição dos objetivos, acompanhamento do progresso do alcance das metas, feedback(quadro de líderes, que exibe o número de ideias de cada participante) e rankings (sistema de pontuação individual e de grupo).A pesquisa demonstrou que os elementos de

Comun. \& Inf., Goiânia, GO, v. 18, n. 1, p. 110-123, jan./jun. 2015 


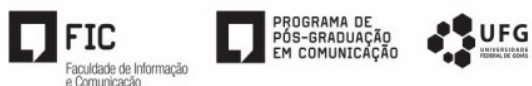

jogosaumentarama geração de ideias. Ou seja, quando aplicados métodos de gamification em sistemas de tomada de decisão,as contribuições entre os usuários aumentaramemtermos de quantidade e qualidade das informações. Além disso, foi observado maior envolvimento dos usuários durante o processo de convergência e refinamento das informações, que geraram melhores resultados para a tomada de decisão (MORADIAN et al., 2014).

\section{DISCUSSÃO DOS RESULTADOS}

Apesar dosestudos sobre o temagamificationseremrecentes, foipossível identificarna literaturaquedesde a década de 80 são realizadas pesquisas relacionadas ao tema de visualização das informações e às influências psicológicas que os métodos visuais com gamification exercem sobre os usuários. O Quadro 2 apresenta um resumo cronológico (colunas)e temático (linhas) dos principais aspectos abordadosnessas áreas,inclusive, com algumas referências de tendências.

Nesta análise cruzada (cronologia x temática),na década de 90,surgem os primeiros conceitos, investimentos de pesquisa e publicações relacionadas à usabilidade e ao design centrado no ser humano. Também são publicados artigos sobre como as apresentações das informações podem influenciar o fluxo e a distribuição das informações para a tomada de decisão.

A partir do ano 2000, as contribuições literárias ampliam em termos de diversidade. Os temas de gestão da informação, design de jogos para visualização da informação, gamification e sistemas de tomada de decisão com gamificationsão tratados por diferentes autores. Vale ressaltar o aumento do número de publicações de casos práticos relacionados ao tema gamification, a partir de 2010. As principais publicações apresentam resultados, principalmente, sobre as influências que os elementos ou mecânicas de jogos podem exercer sobre o comportamento das pessoas em contexto que não são jogos.

Por fim, em termos de tendências para 2015 a 2016, as consultorias Gartner e Research publicam em seus relatórios uma forte estimativa de crescimento em torno do tema gamification. Nesse contexto, entende-se que permaneça a continuidade e o aprofundamento deestudosnos temas tratados neste trabalho: processo decisório e gamification. 
Quadro 2- Abordagem literária por tema x período

\begin{tabular}{|c|c|c|c|c|c|}
\hline & & & Período d & a Publicação & \\
\hline & & $1990-1999$ & $2000-2009$ & $2010-2015$ & $2015-2016$ (previsões) \\
\hline Aborda & em Literária por Tema & $\begin{array}{l}\text { Surgem novos } \\
\text { investimentos das } \\
\text { universidades e pesquisas } \\
\text { relacionadas com a iteração } \\
\text { homem-máquina e conceitos } \\
\text { visuais, tais como, } \\
\text { usabilidade e design } \\
\text { centrado no ser humano. } \\
\text { Neste período são } \\
\text { publicados documentos } \\
\text { sobre a experiências e } \\
\text { relações entre os usuários, } \\
\text { máquina, sistemas de } \\
\text { informação e as influências } \\
\text { que os elementos de jogos } \\
\text { podem exercer sobre os } \\
\text { usuários }\end{array}$ & $\begin{array}{l}\text { São realizadas pesquisas } \\
\text { sobre a gestão e análise } \\
\text { visual das informações } \\
\text { através dos sistemas de } \\
\text { informações e como as } \\
\text { apresentações das } \\
\text { informações podem } \\
\text { influenciar os fluxos das } \\
\text { informações para a } \\
\text { tomada de decisão }\end{array}$ & $\begin{array}{l}\text { Surge o conceito de } \\
\text { gamification e diversas } \\
\text { pesquisas relacionadas a } \\
\text { utilização de métodos de } \\
\text { jogos em contexto que não } \\
\text { são jogos nos mais diversos } \\
\text { segmentos, tais como, na } \\
\text { área de saúde, economia, } \\
\text { entretenimento, tecnologia } \\
\text { da informação, entre } \\
\text { outros. Os principais } \\
\text { estudos demonstram as } \\
\text { influências que os } \\
\text { elementos ou mecânicas de } \\
\text { jogos podem exercer sobre } \\
\text { o comportamento das } \\
\text { pessoas durante a utilização } \\
\text { de processos e sistemas }\end{array}$ & $\begin{array}{l}\text { As consultorias Gartner e } \\
\text { Research apresentam } \\
\text { relatórios de pesquisa que } \\
\text { demonstram uma previsão de } \\
\text { crescimento e utilização de } \\
\text { gamification. As previsões } \\
\text { obtidas através dos estudos } \\
\text { demonstram que as } \\
\text { estimativas são de que em } \\
2015 \text { mais de } 50 \% \text { das } \\
\text { organizações que gerenciam } \\
\text { processos de inovação irão } \\
\text { utilizar métodos de } \\
\text { gamification e que o } \\
\text { crescimento neste mercado é } \\
\text { consistente, considerando } \\
\text { que em } 2016 \text { a tecnologia de } \\
\text { gamification poderá alcançar } \\
\text { a receita de } 2.8 \text { bilhões de } \\
\text { dólares. }\end{array}$ \\
\hline $\begin{array}{l}\text { Gestão da } \\
\text { Informação }\end{array}$ & $\begin{array}{l}\text { Os autores abordam a gestão } \\
\text { da informação como fator } \\
\text { chave em apoio à tomada de } \\
\text { decisão; a importância da } \\
\text { gestão e visualização da } \\
\text { informação através dos } \\
\text { sistemas de análise visual, } \\
\text { para interpretação dos dados } \\
\text { e tomada de decisão }\end{array}$ & $\begin{array}{l}\text {-Davenport e Prusak, 1997; } \\
\text {-Goodman, 1993; } \\
\text {-Wilson, 1999; }\end{array}$ & $\begin{array}{l}\text {-Sawant e Healey, 2007; } \\
\text { - Thomas e Cook, 2005; }\end{array}$ & & \\
\hline
\end{tabular}

Comun. \& Inf., Goiânia, GO, v. 18, n. 1, p. 110-123, jan./jun. 2015 


\section{FIC

Os autores abordam os

conceitos de gamification e

mecânicas de jogos; as

Gamification

tendências da utilização de

gamification; a utilização de

elementos de jogos em

contexto que não jogos.

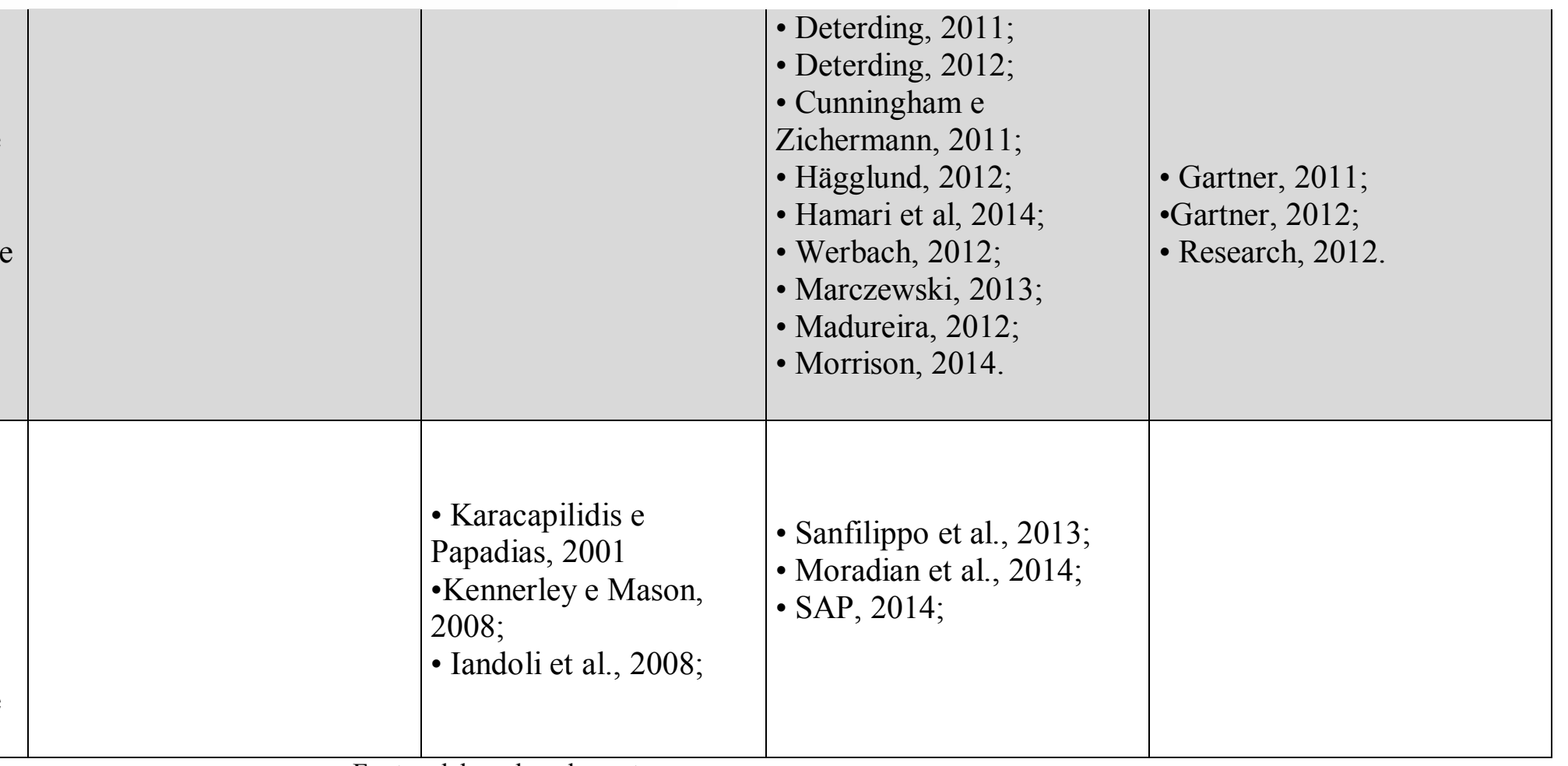

Fonte: elaborado pelos autores. 


\section{CONSIDERAÇÕES FINAIS}

Neste trabalho, foram indicadas as influências que os modelos visuais exercem sobre o comportamento humano em termos das percepções, atenção, motivação, e outros fatores que podem influenciar a criação, troca, distribuição das informações e tomadas de decisões. Além disso, com o excesso de informações e abundância de dados o tema ganha maior relevância, pois a tomada de decisão se torna cada dia mais complexa para as organizações.

Os estudos sobregamificationsão relativamente jovens. Por esse motivo, compreende-se a falta de referências no Brasil nessa área. Da mesma forma, em nível internacional, há uma baixa quantidade e qualidade de estudos sobre a utilização de gamification para a apresentação e visualização de informações para a tomada de decisão.

O trabalho procurou explorar na literatura a importância dos estudos relacionados à apresentação de informações mediante de interfaces visuais atraentes, com o foco em gamification. Foi possível entender por que a utilização de mecânicas e de elementos de jogos em situações convencionais (que não são jogos) apresenta resultados positivos. Nesse sentido, infere-se ser uma boa prática utilizá-los em processos de tomada de decisão, pois podem promover a motivação, aprendizado e facilidade de uso dos indivíduos em sistemas dessa natureza.

De acordo com Hamari et al. (2014), o crescente interesse pelo assunto gamification reflete no contexto acadêmico com o aumento do número de artigos publicados. Entretanto, conforme o autor,há uma carência de entendimentos sobre os tipos de estudos que têm sido realizados nessa área, métodos e tipos de resultados produzidos, e em que circunstâncias. Sugere-se, portanto, como trabalhos futuros, uma maior exploração literária da utilização de gamification aplicados à tomada de decisão, explorando outros resultados nesta área emergente de conhecimento.

Para finalizar, cabe destacar a afirmação "O futuro desse conceito é o melhor possível. Tudo indica que o século XXI será dominado pela diversão e experiência - e o formato que mais se adéqua a essas características é a 'gamificação'."(MADUREIRA,2012).

\section{REFERÊNCIAS}

CODECADEMY. Aprenda a programar de maneira interativa e gratuita. [S. 1.], [201-].

Disponível em: < http://www.codecademy.com>. Acesso em: 31 Ago.2014.

DAVENPORT, T. H.; PRUSAK, L. Information ecology: mastering the information and knowledge environment. USA: Oxford University Press, 1997. 
DETERDING, S. et al. From game design elements to gamefulness: defining "gamification". In: INTERNATIONAL ACADEMIC MINDTREK CONFERENCE: ENVISIONING FUTURE MEDIA ENVIRONMENTS. 15., 2011, Tampere, Finland. Proceedings... New York: ACM, 2011. p. 9-15.

DETERDING, S. Gamification: designing for motivation. Interactions, v. 19, n. 4, p. 14-17, 2012.

EBERT, A.; GERSHON, N. D.; VEER, G. V. der. Human-computer interaction: introduction and overview. KünstlicheIntelligenz, v. 26, n. 2, p. 121-126, 2012.

FERRO, L. S.; WALZ, S. P.; GREUTER, S. Towards personalised, gamified systems: an investigation into game design, personality and player typologies. In: AUSTRALASIAN CONFERENCE ON INTERACTIVE ENTERTAINMENT: MATTERS OF LIFE AND DEATH. 9., 2013, Melbourne, Australia. Proceedings... New York: ACM, 2013. p. 1-6.

GARTNER. Gartner says by 2015, more than 50 percent of organizations that manage innovation processes will gamify those processes. Newsroom,Egham, UK, april 2011. Disponível em:<www.gartner.com/it/page.jsp?id=1629214>. Acesso em: 19 out. 2014.

GARTNER. Gartner says by 2014, 80 percent of current gamified applications will fail to meet business objectives primarily due to poor design. Newsroom, Stamford, Conn. nov. 2012. Disponível em:<www.gartner.com/it/page.jsp?id=2251015>. Acesso em: 19 out. 2014.

GOODMAN, S. K. Information needs for managing decision-making. Records Management Quaterly, v. 27, n. 4, p. 12-23. 1993.

HAMARI, J.; KOIVISTO, J.; SARSA, H. Does gamification work? Aliterature review of empirical studies on gamification. In: HAWAII INTERNATIONAL CONFERENCE ON SYSTEM SCIENCES. 47., 2014, Hawaii, USA. Proceedings...Hawaii, USA, 2014. p. 3025-3034.

HÄGGLUND, P. Taking gamification to the next level: a detailed overview of the past, the present and a possible future of gamification. Student thesis (Degree of bachelor)-UmeåUniversitet, Umeå, 2012.

IANDOLI, L.; KLEIN, M.; ZOLLO, G. Can we exploit collective intelligence for collaborative deliberation? The case of the climate change collaboratorium. MIT Sloan Research Paper, Massachusetts, n. 4675,jul.2008.Disponívelem: <http://ssrn.com/abstract=1084069>. Acesso em: 19 out. 2014.

KARACAPILIDIS, N.; PAPADIAS, D. Computer supported argumentation and collaborative decision making: the HERMES system. Information Systems, v. 26, n. 4, p. 259-277, 2001.

KENNERLEY, M.; MASON, S. The use of information in decision making: literature review for the Audit Commission. Centre for Business Performance, Cranfield School of Management, 2008. Disponível em: $<$ http://archive.audit-

commission.gov.uk/auditcommission/sitecollectiondocuments/AuditCommissionReports/NationalStud ies/Cranfield_Information_use_review.pdf $>$. Acesso em: 04 out. 2014.

KHAN-ACADEMY. A free world-class education for anyone anywhere.2014. Disponível em: <http://www.khanacademy.org/>. Acesso em: 04 Out. 2014.

MADUREIRA, V. Gamificação: mais do que uma tendência, uma estratégia. Marketing Digital,[S. 1.], 2012. Disponível em: <http://imasters.com.br/midia-e-marketing-digital/web-

marketing/gamificacao-mais-do-que-que-uma-tendencia-uma-estrategia/>. Acesso em: 04 out. 2014.

Comun. \& Inf., Goiânia, GO, v. 18, n. 1, p. 110-123, jan./jun. 2015 
MARCZEWSKI, A. Gamification: a simple introduction\& a bit more. [S. 1.]: Kindle edition, 2013.

MILLER, C. et al. Orchestrating data, design, and narrative: information visualization for sense- and decision-making in online learning. International Journal of Cyber Behavior, Psychology and Learning, USA, v. 2, n. 2, p. 1-15, 2012.

MIRANDA, S. V.; STREIT, R. E. Oprocesso de gestão da informação em organizaçõespúblicas.In: ENCONTRO DE ADMINISTRAÇÃO DA INFORMAÇÃO, 1.,2007, Florianópolis. Anais...Santa Catarina: Anpad, 2007. p. 1-17.

MELONI, W.; GRUENER, W. Gamification in 2012. [S. 1.]: M2 Research, 2012. Disponível em: $<$ http://gamingbusinessreview.com/wp-content/uploads/2012/05/Gamification-in-2012-M2R3.pdf $>$. Acessoem: 05 Ago.2014.

MORRISON, B. B.; DISALVO, B. Khan academy gamifies computer science. In: ACM TECHNICAL SYMPOSIUM ON COMPUTER SCIENCE EDUCATION, 45, 2014, New York, USA. Proceedings... New York: ACM, 2014. p. 39-44.

PELLING, N. The (short) prehistory of "gamification"... Funding Startups (\& other impossibilities),[S. 1.], 2011.Disponível em: $<$ http://nanodome.wordpress.com/2011/08/09/the-shortprehistory-of-gamification/>.Acesso em: 04 out.2014.

SANFILIPPO, A. et al. Psychosocial and cultural modeling in human computation systems: a gamification approach. In: MICHELUCCI, P. (ed.). Handbook of human computation. New York: Springer, 2013.

SAP StreamWork Online Help. [S. 1.]: SAP StreamWork. Disponível em: $<$ https://streamwork.com/help/12Sprints.html>.Acessoem: 18 Out. 2014.

SAWANT, A. P.; HEALEY, C. G. Need for perceptual display hierarchies in visualization. XRDS: Crossroads, The ACM Magazine for Students, v. 13, n. 3, 2007.

THOMAS, J. J.; COOK, K. A. Illuminating the path: the research and development agenda for visual analytics. Los Alamitos, USA: IEEE Computer Society, 2005.

WERBACH, K.; HUNTER, D. For the win: how game thinking can revolutionize your business. Philadelphia: Wharton Digital Press, 2012.

WILSON, I. You must remember this. Canada: InstituteonGovernance, 1999.Disponível em: $<$ http://mercury.ethz.ch/serviceengine/Files/ISN/121818/ipublicationdocument singledocument/1435f 0c5-d8dc-499d-8c76-bd2986d741 ee/en/you must remember.pdf>. Acesso em: 19 out. 2014.

CUNNINGHAM, C.; ZICHERMANN, G. Gamification by design: implementing game mechanics in web and mobile apps. Sebastopol: O'Reilly Media, 2011.

Recebido em: 19/10/2014

Aceito em:30/03/2015

Publicado em: 15/06/2015 